\title{
Hybrid Plasma Generation Triggered by a Shunting Arc Discharge Using a Positively Biased Electrode
}

\author{
Ken Yukimura, Member, IEEE, Takafumi Imai, Koichi Takaki, Member, IEEE, and Takashi Ikehata
}

\begin{abstract}
The recent trend in thin film deposition is to prepare ceramic films capable of enduring violent environments, such as corrosive pollutant gases with high temperatures. Different kinds of plasma species existing in the same space are advantageous in order to facilitate a reactive deposition between the gaseous and metallic species. In this paper, we study a carbon shunting arc that triggers a gas discharge using a positively biased electrode. An electrode with a positive voltage of up to $500 \mathrm{~V}$ was set nearby the carbon shunting arc source. Nitrogen, methane, or argon was used as an ambient gas. The induced plasma was generated with a time delay from the moment of the generation of the shunting arc. The electrical and optical characteristics of the discharge were studied. The onset time of the induced plasma as a function of the ambient gas pressure has a Paschen-like V-shaped characteristic. The generated plasma includes both carbon and gaseous species. At the surface of the positively biased electrode, both spectrum emissions of carbon and gaseous species are observed, by which the plasma is confirmed to be mixed. This is based on the spectrum observation in addition to the change in the color of the plasma. A shunting arc is an easy method used in generating a hybrid plasma consisting of metallic (including carbon) and gaseous species. This method promises to efficiently prepare ceramic films by reactive deposition. This method is simple, and both plasma species, i.e., those of metal and gas particles, are easily mixed. The carbon shunting arc plasma plays both the roles of supplying the carbon species and triggering a gaseous plasma as an induced plasma.
\end{abstract}

Index Terms-Arc discharge, emission spectrum, hybrid plasma, Paschen's law, shunting arc discharge.

\section{INTRODUCTION}

$\mathbf{M}$ ETALLIC species have been used not only for ion source applications but also for film deposition by physical vapor deposition or chemical vapor deposition [1], [2]. The recent trend of the thin film preparation is to prepare ceramic films to endure violent environments, such as a corrosive pollutant gas environment with high temperatures. An efficient reactive deposition system under a large volume of plasma is desirable. There are advantages for a hybrid plasma consisting of metallic and gaseous species to enhance the reaction between these species to prepare functional films. In a plasma-based ion implantation and deposition system, 3-D substrates are treated for surface modification [3].

Manuscript received May 9, 2006; revised December 12, 2006.

K. Yukimura and T. Imai are with the Department of Electrical Engineering, Doshisha University, Kyoto 610-0321, Japan (e-mail: kyukimur@ mail.doshisha.ac.jp; dtf0115@mail4.doshisha.ac.jp).

$\mathrm{K}$. Takaki is with the Department of Electronic Engineering, Iwate University, Iwate 020-8551, Japan (e-mail: takaki@iwate-u.ac.jp).

T. Ikehata is with the Department of Electrical and Electronic Engineering, Ibaraki University, Ibaraki 316-8511, Japan (e-mail: tikehata@mx. ibaraki.ac.jp)

Color versions of one or more of the figures in this paper are available online at http://ieeexplore.ieee.org.

Digital Object Identifier 10.1109/TPS.2007.896746
A pulsed plasma may be desirable for nongas ion implantation, because it becomes possible to suppress undesirable surface deposition of the ions by employing a synchronized pulse bias of the substrate. A metallic or a carbon arc is usually triggered by a plasma produced between a pin electrode and a cathode or metal evaporation by an electron beam [4]. No triggering system is desirable for plasma production for the simple construction of the plasma sources.

The shunting arc is one of the plasma production methods for solid-state materials, including metal plasmas, without any external trigger source. The shunting arc is ignited or triggered by self-heating of the rod to increase the vapor pressure and/or to emit thermoelectrons around a rod which is connected to a stored capacitor. A series of articles concerning the shunting arc discharge has been shown in [5]-[13].

Different kinds of plasma species existing in the same space are advantageous for the facilitation of a reactive deposition between the gaseous and metallic species. In this case, both plasma species are activated. When a carbon shunting arc plasma is generated in methane gas, the hydrogen content of the prepared amorphous carbon films is controlled [13]. When a metallic shunting arc is generated in a hydrocarbon gas, the metallic species may be doped in prepared diamond-like carbon films.

In this paper, the gaseous plasma was generated by the shunting arc triggering using a positively biased electrode that is set near a shunting arc source. It is expected that, by this method, species both from the shunting arc discharge plasma and from the gas plasma are mixed by a simple construction of the system; that is, a positively biased electrode is set in the process chamber to make a large potential difference from the plasma source. This accelerates electrons toward the positively biased electrode to induce a gaseous plasma. Thus, the species of the solid materials (in the present case, carbon) are mixed in the gas plasma, which results in making a large volume of the plasma consisting of carbon and gaseous species.

This paper is focused on the electrical and light emission characteristics of the induced plasma, where the ambient gas pressure is varied. It is confirmed that a carbon shunting arc generated in an ambient gas triggers a gaseous plasma in which gaseous and carbon species are mixed.

\section{Production of Shunting ARC}

The shunting arc is a pulsed plasma source for metal or solid materials, and the plasma is produced with a power source which is identical to that used for heating a rod. The rod is set in a chamber in a low-pressure gas or vacuum. Fig. 1 shows a schematic drawing of the shunting arc ignition which develops 




Heating





(a) (b)

(c)

\section{Shunting}

arc



Arcing
Fig. 1. Schematic diagram of generating a shunting arc discharge.

around a solid-state material connected between electrodes. When a current flows through the rod in a low-pressure gas or vacuum and ohmically heats the rod, it results in a vapor pressure increase and a possible emission of thermoelectrons from the rod surface [Fig. 1(a) and (b)]. If the voltage across the rod and the pressure meet the appropriate condition, a breakdown occurs between the electrodes, producing a plasma that contains ions mainly of the material between the electrodes [Fig. 1(c)]. When the impedance of the power supply is low, the breakdown transits to an arc discharge immediately.

Once the arc discharge is established, the discharge current mainly flows through the plasma, and the electrode voltage decreases significantly because of the low impedance of the arc plasma.

The plasma generation around the conductive materials has been known as the shunting arc [14] or the peripheral arc [15]. This arc generation method belongs to so-called "Exploding Wires" [16]-[19]. However, in the previous studies on the exploding wires, the input power to the material was so high that the material was violently exploded or evaporated in a single discharge. On the contrary, in the developed system, we have adjusted the input power such that the material survives for more than 10000 discharge cycles affecting the self-ignition characteristics.

The major advantages of the shunting arc treated in this paper as a plasma source for solid-state materials for industrial applications are summarized as follows.

1) The shunting arc is a self-ignition discharge, requiring no external trigger source, which is common to the conventional arc plasma source.

2) The plasma contains ions mainly of the solid-state material, which is connected to the electrodes, and the relatively long lifetime of the material can be realized by optimizing the discharge condition according to the material.

3) By combining the other plasma sources, such as a radio frequency power source, with the shunting arc sources, a hybrid plasma generation is possible and may efficiently prepare ceramics films consisting of the solid-state materials and gas species.



Fig. 2. Experimental arrangement. Inductance of shunting arc generation circuit: $10.3 \mu \mathrm{H}$ (Circuit 1) and 3.2 $\mu \mathrm{H}$ (Circuit 2).

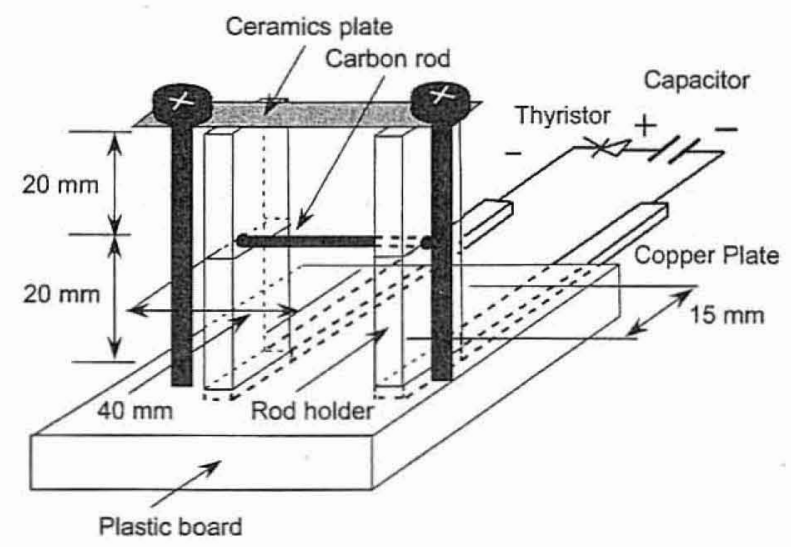

Fig. 3. Schematic diagram of the shunting arc generation unit.

In this paper, we set a positively biased electrode near the shunting arc source to enhance the gas plasma generation triggered by the shunting arc discharge. This plasma generation is done in a very simple way to make a mixed plasma consisting of the metallic (including carbon) and gaseous species.

\section{EXPERIMENT}

Figs. 2 and 3 show schematic diagrams of the experimental arrangement and the shunting arc generation unit, respectively. The chamber was $350 \mathrm{~mm}$ in diameter and 550-mm long. The shunting arc generation unit was on a plastic board, and the rod was connected to the stored capacitor via a thyristor working as a closing switch to initiate the capacitor discharge. A thyristor (DYNEX semiconductor, Type TF94430H, Repetitive peak voltage: $3 \mathrm{kV}$, maximum stationary current: $1.35 \mathrm{kA}$, maximum surge current: $13 \mathrm{kA}$, maximum current rise $(d I / d t)$ : $500 \mathrm{~A} / \mu \mathrm{s}$, maximum on-stage voltage: $2.4 \mathrm{~V}$ ) was used as a closing switch to release the charged energy to heat the carbon rod and to sustain the arc discharge. The charging voltage was set so that the current had a rise time of $20 \%$ of the rated current rise time. The duration of the arc was preprogrammed 




Fig. 4. Schematic diagram of plasma-emission spectrum measurement system.

by the resonance frequency of the capacitance and the circuit inductance.

The carbon rod with a diameter of $2 \mathrm{~mm}$ and a length of $40 \mathrm{~mm}$ was fixed by a pair of carbon holders, as shown in Fig. 3. The holders were fixed to a plastic board using a ceramic plate (1-mm thickness, 10-mm width, and 100-mm length) and two bolts.

A positively biased disc-shaped electrode, with a diameter of $100 \mathrm{~mm}$ and a thickness of $10 \mathrm{~mm}$ facing the shunting arc generation unit, was positioned $100 \mathrm{~mm}$ away from the generation unit. The center of the electrode was the same height as the rod. The positive bias voltage was a charging voltage of $80-\mu \mathrm{F}$ capacitor bank consisting of eight $10-\mu \mathrm{F}$ capacitors, and was varied up to $500 \mathrm{~V}$.

The base pressure for evacuation was $10^{-3} \mathrm{~Pa}$ using a pumping system with two rotary pumps (RP1 and RP2) and a turbo molecular pump (TMP). A polytetrafluoroethylene tube (1/4 in diameter) was set to introduce the gases, such as nitrogen and methane, into the chamber. The gas pressure was adjusted using a mass flow regulator.

Fig. 4 shows a schematic diagram for spectroscopic investigations of the shunting arc discharge and its induced plasma. The output signal from the pulse generator " $A$ " was inputted to the thyristor gate to ignite the shunting arc discharge, and was also inputted to the pulse generator " $\mathrm{B}$ " to run the ICCD (intensified charged couple device, Andor type DH510) to record the emission spectrum intensities. In "B," a delayed pulse was generated to adjust the observation time and the gate time of the ICCD. The output voltage of the signals in the whole system was a TTL level with an amplitude of $5 \mathrm{~V}$.

The visible light emission was observed using a spectroscope with a gated ICCD. The observation point is focused using a collimator lens set in front of the spectroscope. The grating was $150 \mathrm{Gr} / \mathrm{mm}$ (Blaze wavelength: $400 \mathrm{~nm}$ ), and the gate time of the ICCD was fixed at $100 \mathrm{~ns}$. When the emission spectra of the plasma at the surface of the positively biased electrode were observed, the focal point was adjusted on the electrode surface.


Fig. 5. Waveforms of shunting arc discharge current and voltage across the rod of 2-mm diameter and $40-\mathrm{mm}$ length at a nitrogen pressure of $20 \mathrm{~Pa}$.

The current through the circuit was observed using a current transformer (Pearson, type $110 \mathrm{~A}$, sensitivity: $0.1 \mathrm{~V} / 1 \mathrm{~A}$, the rise time: $18 \mathrm{~ns}$, observatory bandwidth: $1 \mathrm{~Hz}-20 \mathrm{MHz}$ ). The voltage across the rod was observed using two voltage probes (Tektronix, type 5100, rated voltage: DC+AC peak of $2.5 \mathrm{kV}$, Frequency bandwidth: DC-250 MHz) and was measured in differential mode. These waveforms are monitored by an oscilloscope (Tektronix, TDS 3034B, Bandwidth: $300 \mathrm{MHz}$, maximum sampling rate: $2.5 \mathrm{GS} / \mathrm{s})$.

We used two circuits to generate the shunting arc discharge plasma. All of the parameters are the same between the two systems, except the circuit parameters. One has a circuit inductance of $10.3 \mu \mathrm{H}$ (Circuit 1), and the other has a circuit inductance of $3.2 \mu \mathrm{H}$ (Circuit 2). Circuit 1 was used in Figs. 5 to 8 , and Circuit 2 was used in Figs. 9 to 13 . In Circuit 1, the capacitance is $20 \mu \mathrm{F}$, with a charging voltage of approximately $1600 \mathrm{~V}$ and with a stored energy of $25.6 \mathrm{~J}$ when charged, which causes a peak current of approximately $1700 \mathrm{~A}$, while in Circuit 2, with a peak current of $1700 \mathrm{~A}$, is obtained with a charging voltage of $1000 \mathrm{~V}$. Although two circuits with different circuit parameters are used, the obtained results are consistent.

\section{RESULTS AND DISCUSSION}

\section{A. Electrical Characteristics of the Carbon Shunting Arc}

Fig. 5 depicts waveforms showing the electrical characteristics of the shunting arc and rod heating. Fig. 5(a)-(c) shows the current through the circuit and voltage across the rod, power, energy, and resistance, respectively. The power is $P=i \times \nu$, where $i$ is current and $\nu$ is voltage. These values are shown in Fig. 5(a). The energy is obtained via integration of the power, 



Fig. 6. Waveforms of current through the shunting arc circuit and its induced current at a positive bias voltage of $160 \mathrm{~V}$ for various nitrogen pressures from 0.74 to $140 \mathrm{~Pa}$.

and resistance is $R=\nu / i$. The nitrogen pressure is $20 \mathrm{~Pa}$, and the capacitor charging voltage is $1600 \mathrm{~V}$.

During the rod heating stage prior to the arc stage, the current and voltage linearly increase with time, and the arc occurs at approximately $1 \mu \mathrm{s}$ at a rod voltage of $350 \mathrm{~V}$. In this case, the thermionic emission current is thought to be less than $0.01 \mathrm{~A} / \mathrm{m}^{2}$ [20], and the consumed energy for the rod heating is approximately $0.07 \mathrm{~J}$, as shown in Fig. 5(b). This is less than $1 / 10000$ of the vaporization energy of the carbon rod that is used in this paper. Thus, the carbon remains even after the repetitive operation of the plasma generation.

In the arc stage, the current shows a sinusoidal behavior, and the voltage is constant with a value of approximately $50 \mathrm{~V}$. The peak power consumed in the arc is approximately $90 \mathrm{~kW}$. Under the experimental conditions, the shunting arc voltage is identical and, thus, is approximately $50 \mathrm{~V}$. The consumed energy during one cycle of the discharge is approximately $2.5 \mathrm{~J}$. During the arc stage, the resistance is constant and is approximately $0.3 \Omega$, as shown in Fig. 5(c).

An induced plasma can be initiated by a positively biased electrode set nearby the shunting arc source. Fig. 6 shows current waveforms of the shunting arc at a nitrogen pressure of $16 \mathrm{~Pa}$ and the induced plasma at pressures from 0.74 to $140 \mathrm{~Pa}$. As shown in Fig. 6(b), the plasma is induced by the shunting arc discharge triggering and the current appears at the positively biased electrode. The initiation time of the induced plasma tends to be increasingly delayed with increased nitrogen pressure. As a common characteristic at the initial stage, the current linearly increases with time and shows a dip, a discontinuity of the current, followed by a sinusoidal shape. It was recognized that no induced plasma current was observed at pressures higher than $140 \mathrm{~Pa}$.

Fig. 7 shows a still photograph of the one-shot discharge at a nitrogen pressure of $16 \mathrm{~Pa}$. The dashed circle in the figure is a field of vision. It is seen in the figure that the induced plasma is generated in the region between the positively biased electrode and the shunting arc source. It is also noticed that the



Fig. 7. Still photograph at a positive bias voltage of $200 \mathrm{~V}$ at a nitrogen pressure of $16 \mathrm{~Pa}$.

plasma consists of two structures. One is a uniform glowlike plasma with a mixed color of blue and red in the right-hand side. Blue and red colors originated from the shunting arc and the nitrogen plasma, respectively. The other is a beamlike plasma with a bright red color and a clear edge, which is generated in the left-hand side. This is seen in the side of the positively biased electrode. Thus, an induced plasma consists of a uniform glowlike plasma and a beamlike plasma.

In order to see the change of the plasma structure, the still photo observation is carried out for varying nitrogen pressures. Fig. 8(a)-(c) shows still photographs at nitrogen pressures of $0.19,44$, and $180 \mathrm{~Pa}$, respectively. The dashed circle in each figure is a field of vision for taking still photographs. In Fig. 8(a), only a uniform plasma is recognized with a mixed color of blue and red, and shows a glowlike plasma. As shown in the figure, the blue-colored plasma is recognized near the positively biased electrode. This shows that the pulsed shunting arc is driven toward the positively biased electrode due to violent generation.

In the case of Fig. 8 (b) at $44 \mathrm{~Pa}$, a beamlike plasma is generated in the half region of the gap in the left-hand side, and its diameter is approximately $12 \mathrm{~mm}$, while the diameter is approximately $20 \mathrm{~mm}$ in Fig. 7. When the pressure is increased, the plasma shrinks. In both cases [Figs. 7 and 8(b)], a beamlike plasma bridges the gap between the positively biased electrode and the edge of the glowlike mixed plasma.

In the case of Fig. 8(c), the diffusion of the plasma is more suppressed, and the gap is not bridged by the plasma. At the surface of the positively biased electrode, a flare is spouted from the surface of the positively biased electrode. A bright spot is seen at the root of the flare on the positively biased electrode. This shows that the flare is originated from the vaporization of the positively biased electrode material. In this pressure region, no induced plasma current is observed.

These phenomena are thought to be closely related to the electrical characteristics. The beamlike plasma shows a transition of the plasma from a uniform glowlike state. At this transition, a dip is made in a current waveform of the induced plasma. However, it should be noticed that the beamlike plasma is partially generated in the side of the positively biased electrode. The glowlike plasma remains in the rest of the space, that is, the beamlike plasma bridges over the space between the positively biased electrode and the edge of the uniform glowlike plasma. 




(a)



(b)



(c)

Fig. 8. Still photographs at a positive bias voltage of $200 \mathrm{~V}$ at a nitrogen pressures of $0.19,44$, and $180 \mathrm{~Pa}$.



Fig. 9. Onset time of the induced plasma as a function of the methane pressure for bias voltages of 200 and $500 \mathrm{~V}$.

The induced plasma is also generated in a methane environment. Fig. 9 shows the onset time of the induced plasma as a function of the methane pressure for positive bias voltages of 200 and $500 \mathrm{~V}$. In both cases, the onset time shows a. V-shaped Paschen-like characteristic curve against the methane pressure, and the minimum onset times are in a pressure between 5 and $15 \mathrm{~Pa}$. Inherently, the characteristic is similar for different gas environments.

The generation of the shunting arc is different from a conventional gas breakdown because of the phenomena that the


Fig. 10. Emission spectra from the carbon shunting arc discharge. Delay time: $15 \mu$ s, air pressure: $0.045 \mathrm{~Pa}$.

degree of rod heating is closely related to the plasma initiation. The V-shaped characteristics of the onset time shown in Fig. 9 show a relationship between a Paschen-like characteristic and the degree of the rod heating.

\section{B. Spectrum Observation}

Fig. 10(a) and (b) shows examples of emission spectra in the absence and in the presence of methane gas, respectively. The observation time of the spectrum in both cases is $12 \mu \mathrm{s}$ after the rod heating. The charging voltage of the $20-\mu \mathrm{F}$ capacitor is $1000 \mathrm{~V}$. Although many emission spectra can be observed, $\mathrm{CII}$ at a wavelength of $426.726 \mathrm{~nm}$ is most significant. When methane gas is introduced, $\mathrm{CH}$ at $431.726 \mathrm{~nm}$ is also observed. This shows that the carbon shunting arc ionizes and/or excites the methane gas particles to make the $\mathrm{CH}$ species. By introducing methane gas, the $\mathrm{CII}$ spectrum intensity is also strengthened.

In the range of the observed wavelength, CI spectra are not observed. CI may be observed in a range from approximately 477 to $505 \mathrm{~nm}$, although their intensities are weak.

Fig. 11(a) and (b) shows a current waveform of the shunting arc discharge and the CII spectrum intensity at $426.726 \mathrm{~nm}$, respectively. The current waveform is observed in the absence of methane gas. However, it was found that the shunting arc current is not largely different under the observed pressure region. It is seen in Fig. 11 that the spectrum intensity generally follows the current waveform. It is also found that the intensity is increased by adding methane gas. The intensity is quickly decreased near end of the shunting arc discharge.

Fig. 12(a) and (b) shows a current waveform of the induced plasma and the temporal behavior of the carbon spectrum intensity at $426.726 \mathrm{~nm}$ for methane gas at a pressure of 1.1 $\mathrm{Pa}$, respectively. The spectrum is observed at the surface of the positively biased electrode. It is seen that the carbon spectrum appears simultaneously with the generation of the glowlike discharge. With increasing current, the intensity becomes greater. There is a dip in the intensity, which may 



Fig. 11. Emission spectra from the carbon shunting arc discharge. (a) Current and (b) emission spectrum intensity (back pressure: $0.045 \mathrm{~Pa}$ ).
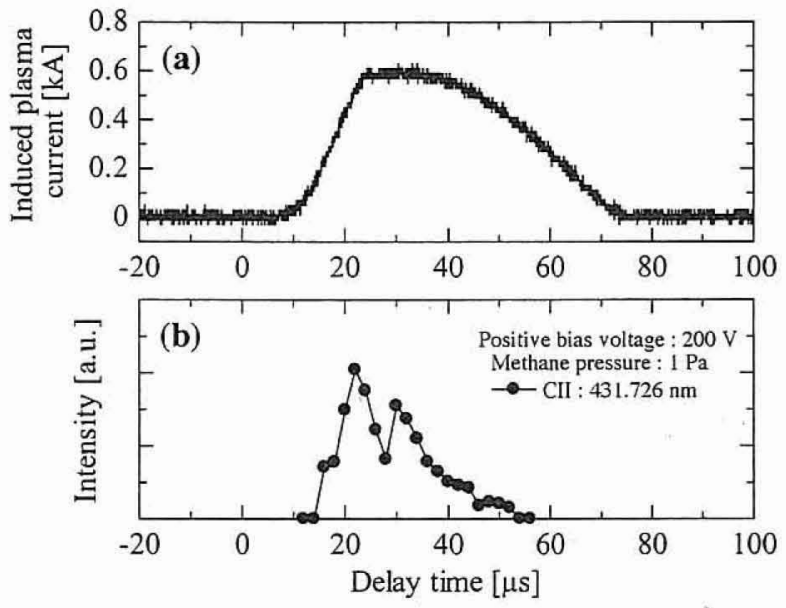

Fig. 12. Temporal behavior of the induced plasma current and the spectrum intensity of carbon at a wavelength of $431.726 \mathrm{~nm}$ at a methane pressure of $1 \mathrm{~Pa}$ for a positive bias voltage of $200 \mathrm{~V}$.

correspond to the current dip. The induced plasma is seen until approximately $80 \mu \mathrm{s}$, while the spectrum intensity is clearly recognized until approximately $60 \mu \mathrm{s}$, which is approximately $20 \mu$ s shorter than the lifetime of the induced plasma. This is probably due to the diffusion of the mixed plasma consisting of a carbon shunting arc plasma and the induced methane plasma.

The mixing of the shunting arc and the induced plasma is checked by the generation of a shunting arc in an argon gas environment. Fig. 13 shows the spectra of the induced plasma observed at the surface of the positively biased electrode at $200 \mathrm{~V}$ for an argon pressure of $0.7 \mathrm{~Pa}$. The observation time was $28 \mu$ s starting from the rod heating. The emissions from both carbon and argon particles are observed. This means that argon, as an ambient gas, which is ionized in the induced plasma, is triggered by the shunting arc discharge. It is noticed that the carbon species generated by the shunting arc discharge arrive at the positively biased electrode due to the driving by a pulsed plasma generation. Thus, it is confirmed that a hybrid plasma consisting of argon and carbon species is made, and the shunting arc and its induced plasma are mixed.



Fig. 13. Emission spectra of the argon plasma induced by the carbon shunting arc discharge (Argon pressure: $0.7 \mathrm{~Pa}$, and positive bias voltage: $200 \mathrm{~V}$ ). The emission was observed at the surface of the positively biased electrode.

\section{CONCLUSION}

The carbon shunting arc triggers a gas plasma using a positively biased electrode. It is confirmed that both plasma species are mixed. The state of mixing is dependent on the ambient gas pressure.

In the low-pressure region of under $1 \mathrm{~Pa}$, a glowlike plasma is observed at the gap between the positively biased electrode and the shunting arc source, and its color is mixed with blue from the shunting arc and red from the nitrogen plasma. Carbon species generated in the shunting arc discharge arrive at the positively biased electrode.

In a middle pressure region between 1 and approximately $100 \mathrm{~Pa}$, a beamlike arc plasma partially bridges the gap between the positively biased electrode and the edge of the glowlike discharge. There is a discontinuity of the current waveform during the induced plasma. The current discontinuity corresponds to the change in the emission spectrum intensity of the induced plasma. The current discontinuity and the current dip may be closely related to each other; that is, the induced plasma is first generated as a uniform glowlike plasma; it is followed by a beamlike plasma partially bridging the gap, while a glowlike discharge remains in the rest of the region.

In a high-pressure region of over approximately $100 \mathrm{~Pa}$, the diffusion of the shunting arc is suppressed, and no induced plasma is generated. A flare having a root on the positively biased electrode is observed, but no bridging over phenomenon.

From the emission spectra from the shunting arc discharge plasma and its induced plasma, it is confirmed that the shunting arc triggers the gas discharge and ionizes the gas species. The onset time of the induced plasma has a V-shaped Paschen-like characteristic against the ambient pressure.

\section{REFERENCES}

[1] I. G. Brown, "Vacuum arc ion sources," Rev. Sci. Instrum., vol. 65, no. 10, pp. 3061-3081, Oct. 1994.

[2] R. L. Boxman, S. Goldsmith, and A. Greenwood, "Twenty-five years of progress in vacuum arc research and utilization," IEEE Trans. Plasma Sci., vol. 25, no. 6, pp. 1174-1186, Dec. 1997.

[3] B. P. Wood, D. J. Rej, A. Anders, I. G. Brown, R. J. Faehl, S. M. Malik, and C. P. Munson, "Fundamentals of plasma immersion ion implantation," in Handbook of Plasma Immersion Ion Implantation and Deposition, A. Anders, Ed. New York: Wiley, 2000, ch. 4.

[4] R. Dougal, I. G. Brown, and S. K. Händel, "Pulsed power applications," in Handbook of Vacuum Science Technology-Fundamentals and 
Applications-, R. L. Boxman, P. J. Martin, and D. M. Sanders, Eds. Park Ridge, NJ: Noyes, 1995, ch. 9.

[5] K. Yukimura, R. Isono, T. Monguchi, and K. Yoshioka, "Pulsed metal ion source by triggerless shunting arc discharge," J. Vac. Sci. Technol. B, Microelectron Process Phenom., vol. 17, no. 2, pp. 871-874, Mar. 1999.

[6] K. Yukimura, K. Yoshioka, and Y. Tani, "Carbon shunting arc and its induced plasma," Rev. Sci. Instrum., vol. 71, pt. II, no. 2, pp. 1184-1186, Feb. 2000.

[7] K. Yukimura and Y. Tani, "Generation of triggerless silicon shunting plasma and ion extraction," Surf. Coat. Technol., vol. 142-144, pp. 388-391, Jul. 2001.

[8] K. Yukimura and S. Masamune, "Shunting arc plasma generation over a wide range of ambient pressure and ion extraction," Rev. Sci. Instrum., vol. 73, pt. II, no. 2, pp. 860-862, Feb. 2002.

[9] K. Yukimura and S. Masamune, "Shunting arc plasma generation and ion extraction," Surf. Coat. Technol., vol. 156, no. 1-3, pp. 31-37, Jul. 2002.

[10] K. Takaki, S. Mukaigawa, T. Fujiwara, M. Kumagai, and K. Yukimura, "Ion extraction from carbon shunting arc plasma," Appl. Phys. Lett., vol. 82, no. 16, pp. 2583-2585, Apr. 2003.

[11] K. Takaki, R. Hasegawa, S. Nakamura, S. Mukaigawa, T. Fujiwara, M. Kumagai, and K. Yukimura, "Self-magnetic drive of a shunting arc," Surf. Coat. Technol., vol. 186, no. 1/2, pp. 182-186, Aug. 2004.

[12] K. Yukimura, M. Kumagai, K. Takaki, R. Hasegawa, S. Nakamura, S. Mukaigawa, and T. Fujiwara, "High deposition rate of amorphous carbon film using a magnetically driven shunting arc discharge," Surf. Coat. Technol., vol. 196, no. 1-3, pp. 203-206, Jun. 2005.

[13] K. Yukimura, K. Ego, K. Takaki, S. Mukaigawa, and T. Fujiwara, "Amorphous carbon film deposition by PBII\&D using shunting arc discharge," Nucl. Instrum. Methods Phys. Res. B, Beam Interact. Mater. At., vol. 242, no. 1/2, pp. 321-323, Jan. 2006.

[14] S. A. Bordzilovskii, S. M. Karakhanov, and V. V. Polyudov, "Electron emission in pulsed heating of a wire," Sov. Phys. Tech. Phys., vol. 18, no. 9, pp. 1251-1254, Mar. 1974

[15] F. D. Bennett, H. S. Burden, and D. D. Shear, "Correlated electrical and optical measurements of exploding wires," Phys. Fluids, vol. 5, no. 1, pp. 102-113, Jan. 1962.

[16] W. G. Chace and H. K. Moore, Eds., Exploding Wires, vol. 1. New York: Plenum, 1959.

[17] W. G. Chace and H. K. Moore, Eds., Exploding Wires, vol. 2. New York: Plenum, 1961.

[18] W. G. Chace and H. K. Moore, Eds., Exploding Wires, vol. 3. New York: Plenum, 1964.

[19] W. G. Chace and H. K. Moore, Eds., Exploding Wires, vol. 4. New York: Plenum, 1967.

[20] A. Anders, J. Pelletier, D. M. Goebel, B. P. Wood, I. G. Brown, W. Ensinger, and M. Tuszewski, "Plasma sources," in Handbook of Plasma Immersion Ion Implantation and Deposition, A. Anders, Ed. New York: Wiley, 2000, ch. 7.



Ken Yukimura (M'99) received the B.Eng., M.Eng., and Dr.Eng. degrees in electrical engineering from Doshisha University, Kyoto, Japan, in 1970, 1972, and 1977 , respectively.

From 1977 to 1979 , he worked with the Faculty of Engineering, Doshisha University, as a Research Assistant, and between 1986 and 1992, as an Associate Professor with Doshisha University. Since 1992, he has been a Professor at Doshisha University. His current research interests include pulsed power technology such as the development of a new plasma source for the solid materials, pulsed ion technology such as 3-D ion implantation technology, and exhaust gas processing in the atmospheric pressure such as decomposition of NOx. He has research experiences of the exploding wire phenomena, excimer laser and vacuum ultraviolet emission technology, ion technology and surface modification technology by plasmas at atmospheric pressure.

Dr. Yukimura was a Cochair person of the Fifth International Workshop on Plasma-based Ion Implantation (Kyoto, Japan, December 1999) and is a Guest Editor of special issues relevant to Plasma-Based Surface Modification and Treatment Technologies in the IEEE TRANSACTIONS ON PLASMA SCIENCE.



Takafumi Imai received the B.Eng. degree in electronic engineering from Doshisha University, Kyoto, Japan, in 2005. Currently, he is working toward the Master degree in electrical engineering at Doshisha University.



Koichi Takaki (M'99) was born in Fukuoka, Japan, on October 16, 1963. He received the B.Eng., M.Eng., and Dr.Eng. degrees in electrical engineering from Kumamoto University, Kumamoto, Japan, in 1986,1988 , and 1995 , respectively.

In 1989 and 1993, he was a Research Associate and Lecturer, respectively, with Oita National College of Technology. In 1996 and 2000, he was a Research Associate and Associate Professor, respectively, with the Department of Electrical and Electronics Engineering, Iwate University, Morioka, Japan. During 2000-2001, he was a Visiting Scientist with McMaster University, Hamilton, ON, Canada. His current research interests include basic and applied research in various aspects of gaseous discharge and pulsed power.



Takashi Ikehata received the Ph.D. degree in physics from University of Tsukuba, Tsukuba, Japan, in 1983.

In 1983, he joined Ibaraki University, Hitachi, Japan, as a Research Associate, where he is currently a Professor of plasma science in the Institute of Applied Beam Science. His current research interests include plasma and ion beam sources for material processing and analysis, field-emitter electron sources and their applications, and the ultratrace analysis of environmental pollutants by laser mass spectrometry

Dr. Ikehata is a member of the Institute of Electrical Engineers of Japan, the Japan Society of Applied Physics, and the Japan Society of Plasma Science and Nuclear Fusion Research. 